\title{
Contribution to the study and analysis of microwave bandpass filter based on circular Split Rings Resonators
}

\author{
Mohammed Berka ${ }^{1}$, Zoubir Mahdjoub ${ }^{2}$, Tarik Bendimered ${ }^{3}$ \\ ${ }^{1}$ (Department of Electrical Engineering / University of Mascara, Algeria) \\ ${ }_{2}^{2}$ (Department of Electronic / University of Sidi Bel Abbes, Algeria) \\ ${ }^{3}$ (Department of Electronic / University of Tlemcen, Algeria)
}

\begin{abstract}
The purpose of this article is the analysis and design of a new structure of the bandpass filter, the analysis of the composite structure is made from a bandpass filter using microstrip resonators connected in parallel that are associated with a circular Split Rings Resonators metamaterials present a negative permeability (SRR) to create an environment of retro-propagation of waves. The behavior of the composite filter is also bandpass but around the resonance frequency of (SRR), which is inferior to the center frequency of the first band-pass filter, which allows a good operation of the composite filter in the stop-band of the first bandpass filter with resonators coupled in parallel. Since there's no scientific or experimental result in the literature for this kind of complicated structure, it was necessary to perform simulations using CST MICROWAVE STUDIO.
\end{abstract}

Keywords: bandpass filter, coupled resonator, CST MICROWAVE STUDIO, permeability, SRR metamaterials.

\section{Introduction}

Modern telecommunications are moving towards "wholes wireless", while microwave applications are introduced to meet this need. Among these applications, noting the design of microwave filters, including filters using microstrip resonators coupled in parallel [1] which are widely used because of its low economic cost and also small dimensions during their realizations. The performance of this kind of filter is enhanced with new physical devices that are metamaterials.

Metamaterials are artificial pseudo-homogeneous structures with electromagnetic properties not found in nature; an analysis of this kind of structure was originally made by the Russian physicist Victor Veselago [2]. The physical properties of metamaterials are represented by a permittivity $\varepsilon$ and permeability $\mu$ simultaneously negative [3-4].

To demonstrate the influence of metamaterials on the quality of microwave filters, we propose in this paper a new structure that includes a coupled resonators filter associated in parallel with metamaterials in Split Ring Resonators (SRR) which are chosen from their circular form and magnetic activity [5], their dimensions are selected to resonate in the $\mathrm{X}$ band [8-12] GHz. The dimensions of the filter with resonators coupled in parallel are selected to achieve a center frequency of the bandpass around $21.4 \mathrm{GHz}$ which is much higher than the resonance (SRR) in the $\mathrm{X}$ band. The choice of number of (SRR) and their position above the parallel coupled resonator filter is very important to allow the composite structure to function outside the passband of the parallel coupled resonators filter.

\section{Methodology}

2.1. Analysis of parallel-coupled resonators filter.

Figure 1 shows the band-pass filter consists of a set of five $(n=5)$ microstrip parallel coupled resonators. Each resonator has a length $(\lambda / 2)$ at the center frequency $f_{0}$ of the bandpass filter and coupled to the other of $(\lambda / 4)$. We design this kind of filter in a Tchebyshev filtering function, used the synthesis of Matthaei that allows us to determine the impedances even and odd mode for each quarter of symmetric coupled lines, these impedances are also calculated from the elements $\left(g_{i}\right)$ of the Tchebyshev lowpass prototype and depending on ripple $\left(r_{p}\right)$ in the passband of this filter.

For a filter of $\mathrm{n}$ resonators, Matthaei synthesis can be summarized in [6]:

-The parameters $g_{i}(i=1,2, \ldots . ., n)$ of the standard prototype.

$g_{0}=1, g_{1}=\frac{2 a_{1}}{\gamma}$

$g_{i}=\frac{4 a_{i-1} a_{i}}{b_{i-1} b_{i}}$, for $i=2, \ldots, n$

$g_{n+1}=1 \quad ; \mathrm{n}$ odd

$g_{n+1}=\tan ^{2}\left(\frac{\beta}{4}\right)$

even

(4) 


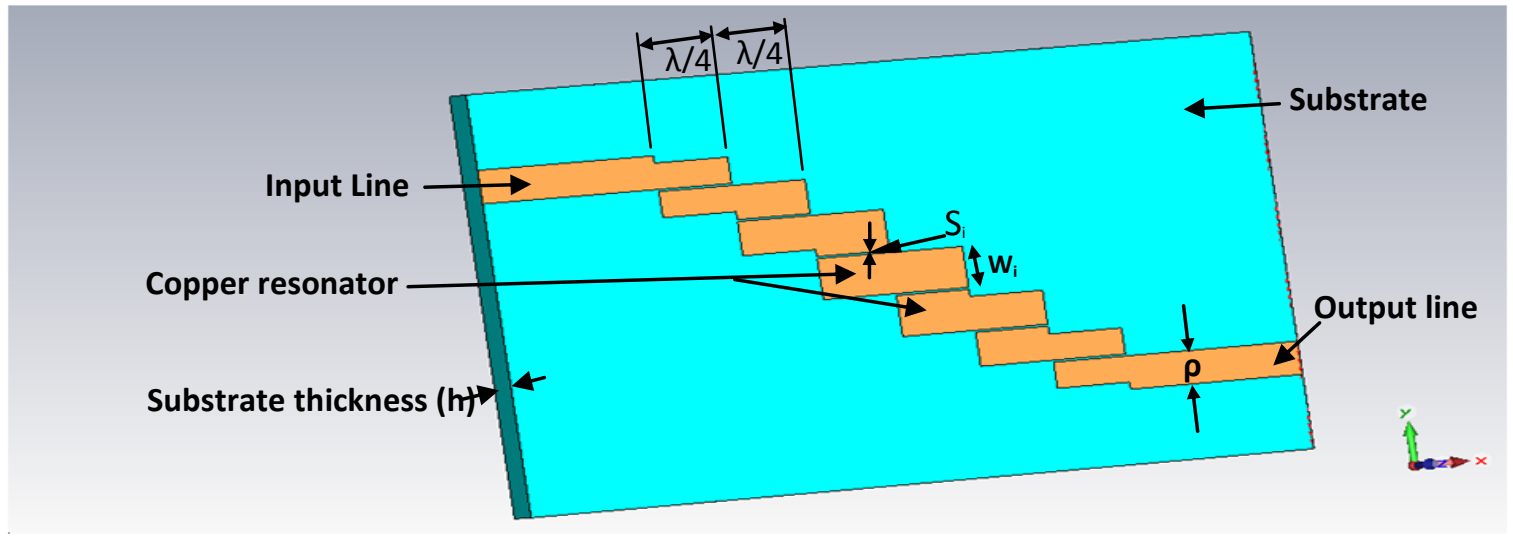

Figure 1. Bandpass filter with five parallel-coupled microstrip resonators

$a_{i}=\sin \left[\frac{(2 i-1) \pi}{2 n}\right]$

for

$i=1$,

$2, \ldots$.

$n$

(5)

$b_{i}=\gamma^{2}+\sin ^{2}\left(\frac{i \pi}{n}\right)$, for $i=1,2, \ldots ., n$

Where: $\quad \gamma=\sin \left(\frac{\beta}{2 n}\right)$

and

(7)

-The admittance inverter parameters are:

$J_{0,1}=J_{n, n+1}=\frac{Z_{0}}{\sqrt{g_{0} g_{1}}}=\frac{Z_{0}}{\sqrt{g_{0 n} g_{n+1}}}$

$J_{i, i+1}=\frac{z_{0}}{\sqrt{g_{i} g_{i+1}}}$

; $\quad i=1,2, \ldots \ldots$

$n-1$

(9)

-The impedances of resonators for even and odd modes are:

$Z_{0 e}(1)=Z_{0 e}(n+1)=Z_{0}[1+A]$

for even modes

(10)

$Z_{0 o}(1)=Z_{0 o}(n+1)=Z_{0}[1-A]$

for odd modes.

(11)

Where:

$$
A=\frac{R}{\sqrt{0.5 \tan \theta_{1}+R^{2}}}
$$

and

$R=\frac{J_{0,1}}{Z_{0}}$

(12)

$Z_{0 e}(i+1)=q Z_{0}\left(M_{i, i+1}+\frac{J_{i, i+1}}{Z_{0}}\right)$

for

even modes;

$i=1,2, \ldots ., n-1$

(13)

$Z_{0 o}(i+1)=q Z_{0}\left(M_{i, i+1}-\frac{J_{i, i+1}}{Z_{0}}\right)$

for odd modes;

$i=1,2, \ldots \ldots, n-1$

(14)

Where: $\left\{\begin{array}{l}q=\frac{1}{\frac{\tan \theta_{1}}{2}+\left(\frac{J_{0,1}}{Z_{0}}\right)^{2}} \text { and } M_{i, i+1}=\sqrt{\left(\frac{J_{i, i+1}}{Z_{0}}\right)^{2}+\frac{\tan ^{2}\left(\theta_{1}\right)}{4}} \\ \theta_{1}=\frac{\pi}{2}\left(1-\frac{B}{2}\right) \text { and } B=\frac{f_{2}-f_{1}}{f_{0}}\end{array}\right.$

-Dimensions of microstrip resonators:

$\frac{W}{h}=\frac{2}{\pi(\alpha-1)}-\frac{2}{\pi} \ln (2 \alpha-1)+\left(\frac{\varepsilon_{r}-1}{\pi \varepsilon_{r}}\right)\left[\ln (\alpha-1)+0.293-\frac{0.517}{\varepsilon_{r}}\right]$

where: $\alpha=\left\{\begin{array}{cl}\frac{60 \pi^{2}}{Z_{0}^{\prime} \sqrt{\varepsilon_{r}}} \text { avec } Z^{\prime}{ }_{0}=\frac{Z_{0 e}}{2} \text { for even modes. } \\ \frac{60 \pi^{2}}{Z^{\prime \prime}{ }_{0} \sqrt{\varepsilon_{r}}} \text { avec } Z^{\prime \prime}{ }_{0}=\frac{Z_{0 o}}{2} \quad \text { for odd modes. }\end{array}\right.$ 
$\frac{S}{h}=\frac{2}{\pi} \cosh ^{-1}\left[\frac{\cosh \left[\frac{\pi}{2}\left(\frac{W}{h}\right)_{e}\right]+\cosh ^{-2}\left[\frac{\pi}{2}\left(\frac{W}{h}\right)_{o}\right]}{\cosh \left[\frac{\pi}{2}\left(\frac{W}{h}\right)_{o}\right]-\cosh ^{-2}\left[\frac{\pi}{2}\left(\frac{W}{h}\right)_{e}\right]}\right]$

(19)

Where $\left(Z_{0}=50 \Omega\right)$ present the characteristic impedance of the input and output lines and B present the desired fractional bandwidth.

\subsection{Basic of Circular (SRR):}

The circular (SRR) are periodic structures with a low period vis-à-vis wavelength. The Yablonovitch works [7] show that periodic structures can be classified according to their constitution which may be metallic, dielectric or metallo-dielectric. The (SRR) circulars represent metamaterials with negative permeability ( $\mu_{\text {eef }}$ $<0$ ), they are dimensioned for operation in band $\mathrm{X}$ [8-12] GHz, enabling resonance at a frequency where the wavelength of the vacuum is much greater than its diameter. The geometric properties of a (SRR) are shown in Figure 2.

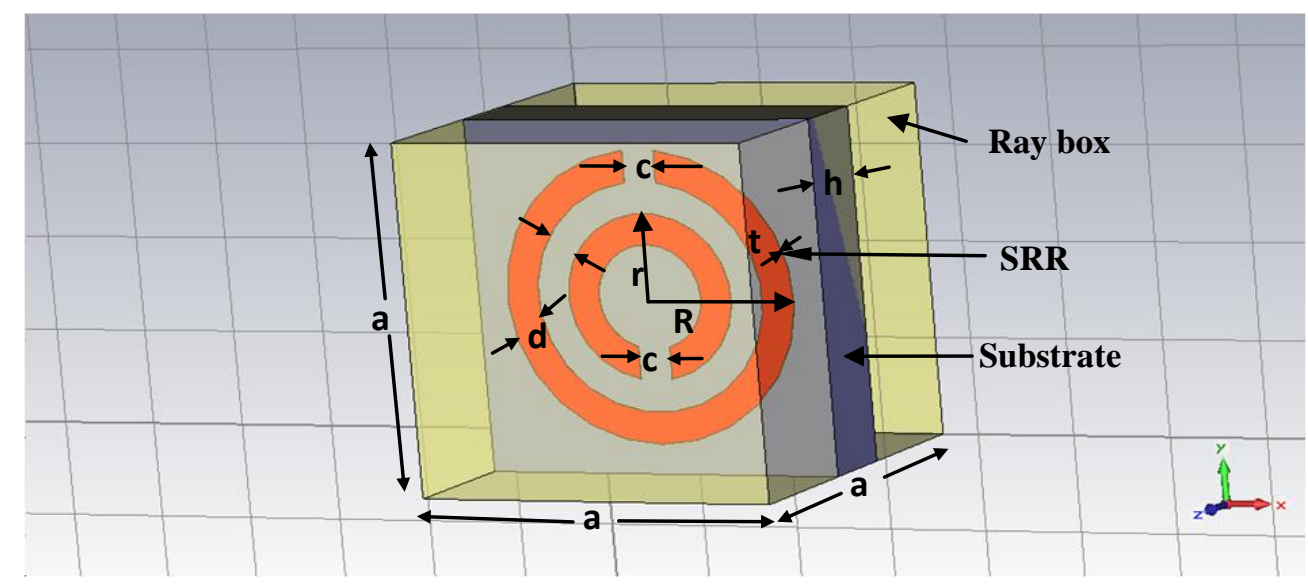

Figure 2. (SRR) geometric shape

-The geometric parameters of circular (SRR) are:

$a=$ period of the structure, $b=$ distance between the two rings.

$\mathrm{c}=$ width of the rings cut, $\mathrm{d}=$ width of the tow rings.

$(\mathrm{R}, \mathrm{r})$ represent respectively, the external radius of the large and small rings (SRR).

$\mathrm{h}=$ thickness of the substrate and $\mathrm{t}=$ thickness of the engrave ring on the substrate

-The physical parameters of (SRR) are:

$\varepsilon_{\mathrm{r}}=$ relative permittivity of the substrate.

Conductor tracks that form two rings (copper).

\section{Simulation results in CST MICROWAVE STUDIO}

\subsection{Filter with parallel-coupled resonators.}

On a Alumina $(96 \%)$ substrate $\left(\varepsilon_{\mathrm{r}}=9.4\right)$ with a loss factor $(\tan \delta=0.0004)$ and thickness $(\mathrm{h}=$ $0.635 \mathrm{~mm})$, the design of bandpass Tchebyshev filter is made using five $(\mathrm{n}=5)$ microstrip resonators in a copper with thickness $(\mathrm{t}=5 \mu \mathrm{m})$. Dimensions of the filter are obtained to have a center frequency around $21.4 \mathrm{GHz}$, the width of the input and output lines $(\mathrm{h}=\rho)$ are selected to obtain an adaptation to $50 \Omega$ and the $\left(\mathrm{r}_{\mathrm{p}}=0.5 \mathrm{~dB}\right)$ in the passband

-The geometric characteristics of this filter are summarized in Table 1.

\begin{tabular}{|c|l|l|l|l|l|l|l|}
\hline $\boldsymbol{i}$ & $\boldsymbol{g}_{\boldsymbol{i}}$ & $\boldsymbol{Z}_{\boldsymbol{\theta}_{e}(\boldsymbol{\Omega})}$ & $\boldsymbol{Z}_{\boldsymbol{o}_{\boldsymbol{o}}(\boldsymbol{\Omega})}$ & $\boldsymbol{W / h}$ & $\boldsymbol{S} / \boldsymbol{h}$ & $\boldsymbol{W}(\boldsymbol{m m})$ & $\boldsymbol{S}(\mathbf{m m})$ \\
\hline 1 & 1.7058 & 81.3009 & 18.6991 & 0.7839 & 0.0635 & 0.4977 & 0.0403 \\
\hline 2 & 1.2296 & 61.2311 & 15.0976 & 1.0211 & 0.0604 & 0.6483 & 0.0383 \\
\hline 3 & 2.5408 & 54.7148 & 16.8921 & 1.2308 & 0.0601 & 0.7815 & 0.0381 \\
\hline 4 & 1.2296 & 54.7148 & 16.8921 & 1.2308 & 0.0601 & 0.7815 & 0.0381 \\
\hline 5 & 1.7058 & 61.2311 & 15.0976 & 1.0211 & 0.0604 & 0.6483 & 0.0383 \\
\hline 6 & 1.0000 & 81.3009 & 18.6991 & 0.7839 & 0.0635 & 0.4977 & 0.0403 \\
\hline
\end{tabular}

Table 1. Geometric characteristics of the bandpass filter 
-Figure 3 shows in the frequency range [0-40] GHz the results of frequency responses of Tchebyshev filter with parallel-coupled resonators represented in Figure1 which have geometrical characteristics summarized in Table1. Figure 3 shows the behavior passband of Tchebyshev filter for the approximate center frequency $\left(f_{0}=\right.$ $21.4 \mathrm{GHz}$ ), where bandpass has five waves which justifies the number of resonators.

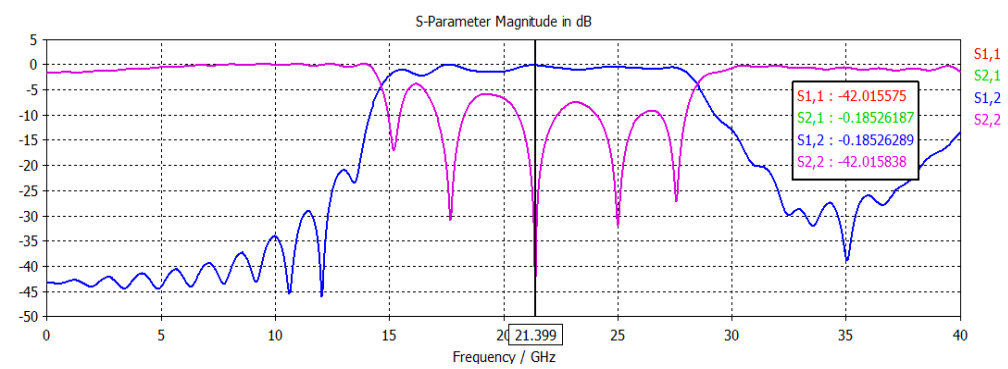

Figure 3. [S] Parameters of the Tchebyshev filter

\section{2. (SRR) behavior.}

The Split Rings Resonators used for the design of the composite filter are dimensioned such that it has a resonant frequency in the $\mathrm{X}$ band [8-12] GHz. (SRR) Parameters are:

$\mathrm{a}=1.1125 \mathrm{~mm}, \mathrm{~b}=\mathrm{c}=\mathrm{d}=0.1 \mathrm{~mm}, \mathrm{R}=0.4562 \mathrm{~mm}, \mathrm{r}=0.2562 \mathrm{~mm}$

Alumina-substrate $\left(\varepsilon_{\mathrm{r}}=9.4\right)$ has the same thickness of the filter substrate $(\mathrm{h}=0.635 \mathrm{~mm})$

-Thickness of copper rings $(\mathrm{t}=5 \mu \mathrm{m})$.

-The simulation of (SRR) is made in CST MICROWAVE STUDIO, using a box of radiation of the same period $(\mathrm{a}=1.1125 \mathrm{~mm})$.

-Figure 4 provides in the $\mathrm{X}$ band [8-12] GHz, the results simulation of (SRR), it shows a stopband behavior from the transmission coefficient $\left(S_{21}\right)$, which has a minimum $(-18 \mathrm{~dB})$ in the frequency resonance $f_{r}=9.75$ $\mathrm{GHz}$.

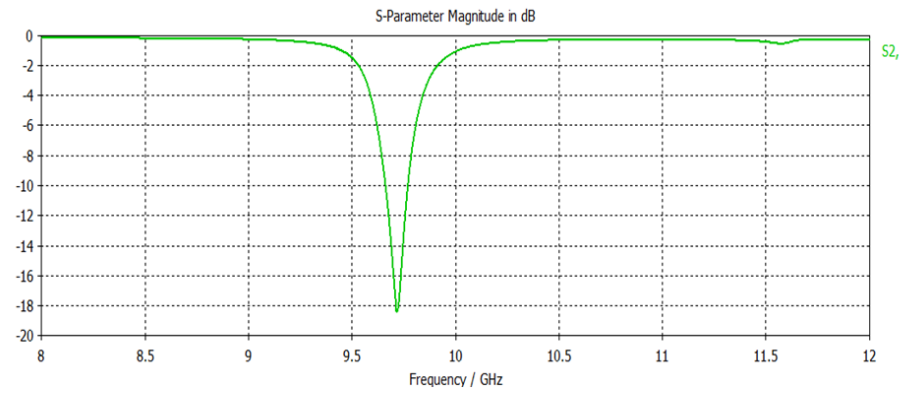

\subsection{Composite filter.}

Figure 4. Transmission coefficient of circular (SRR)

For the composite filter, we associate the (SRR) to the bandpass Tchebyshev filter. The number of (SRR) and their positions are shown in Figure 5.

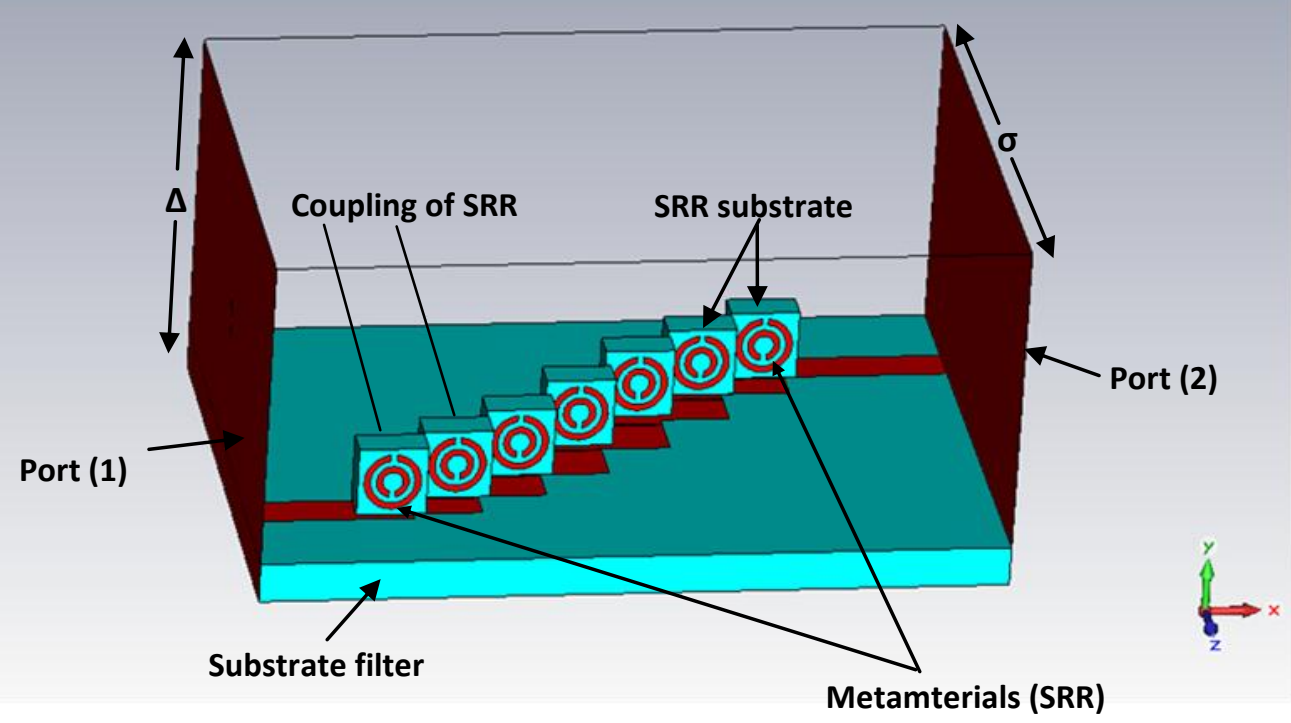

Figure 5. Composite filter 
- To supply (SRR), we place it just above the input and output lines such that the cuts of the rings are perpendicular to the microstrip resonators and they are upwardly for the outer ring and to the low for the internal ring in order to provide maximum electromagnetic coupling between these (SRR) and microstrip resonators. In CST MICROWAVE STUDIO we feed the overall filter by two supply ports which have these $\operatorname{sizes}(5 \mathrm{~h} \leq \Delta \leq 10 \mathrm{~h})$ and $(0 \leq \sigma \leq 6 \mathrm{~h})$. The (SRR) are coupled in a vertical manner relative to resonator microstrip and a parallel manner between them to ensure a good electromagnetic coupling. The number of (SRR) must be adequate to the number of microstrip resonators, so that $(n=5)$ resonators, it takes seven (SRR) for providing electromagnetic coupling to each resonator and for supplying the (SRR).

- Each (SRR) substrates has a height which is the same as the period value $(\mathrm{a}=1.1125 \mathrm{~mm})$ and has a thickness which is the same value of $\left(\mathrm{W}_{1}=0.4977 \mathrm{~mm}\right)$.

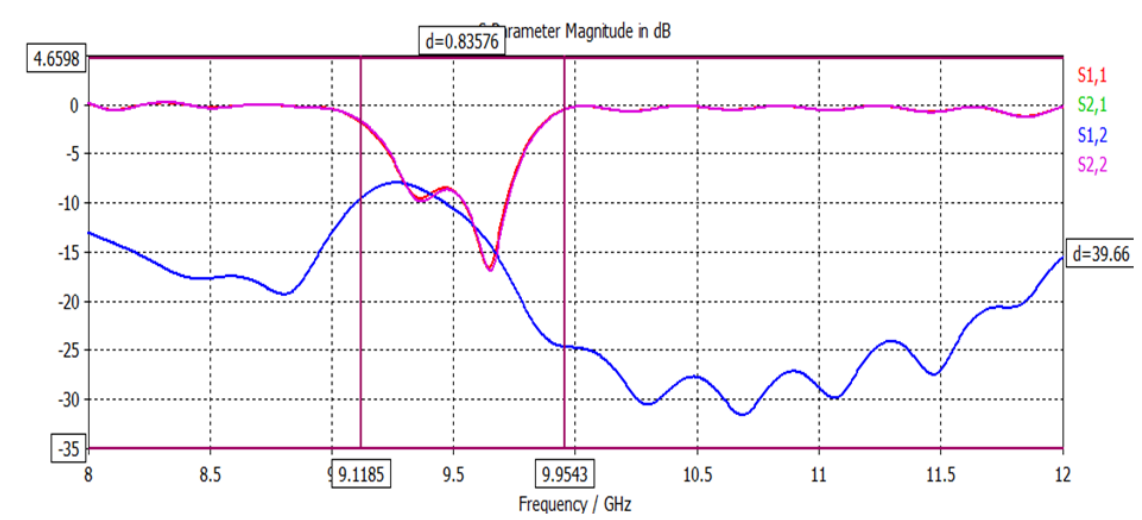

Figure 6. Parameters [S] of the composite filter

Figure 6 shows that the behavior of the filter composed by microstrip resonators coupled in parallel and circular (SRR) is a passband too, with a resonance around the approximate center frequency $\mathrm{f}_{0}{ }^{\prime}=9.54 \mathrm{GHz}$ instead of $21.4 \mathrm{GHz}$ and for bandwidth [9.1185 - 9.9543] GHz. This behavior shows the influence of metamaterials on microwave Tchebyshev filter.

\section{Conclusion}

A new structure of microwave filters have been proposed, a combination of circular Split Rings Resonators metamaterials with Tchebyshev bandpass filter has enabled this filter to change its characteristics thus their operation in the stopband of the first filter. The influence of metamaterials is shown in the quality of filters, such as minimizing insertion loss and ripples in the new bandpass for the Tchebyshev filter. This influence is shown also in the conservation of overall filter behavior but for other frequency range.

\section{Acknowledgments}

The authors would like to thank Pr. Tarik Bendimered for his support. They also thank him for his availability concerning the partnership with the Telecommunications Laboratory of Tlemcen (TLT).

\section{References}

[1] S. B .Cohn, Parallel-coupled transmission-line-resonator filters, IRE Transactions on Microwave Theory and Techniques, vol. 6 , no. 2, 223-231, 1998.

[2] V. G. Veselago, The electrodynamics of substance with simultaneously negative values of $\varepsilon$ and $\mu$, Soviet physics USPEKHI, vol.10, no.14, 509-514, January-February 1968

[3] C. Caloz, T. Itoh, Electromagnetic metamaterials transmission line theory and microwave applications, John Wiley \& Sons, 2006.

[4] G. V. Eleftheriades, Enabling RF/Microwave Devices using Negative-Refractive-Index Transmission Line Metamaterials, Radio Science Bulletin No 132, Mars 2005.

[5] J. B. Pendry, A.J.Holden, D.J.Robbins and W.J.Stewart, Magnetism from conductors and enhanced nonlinear phenomena, IEEE Transmission on Microwave Theory and Techniques, vol. 47, no.11, 2075-2084, November 1999.

[6] G. L. Matthaei, L. Young, and E. M. T. Jones, Microwave filters, impedance-matching networks, and coupling structures, Mc Graw-Hill Book Company, 1964.

[7] E. Yablonovitch, Photonic band-gap structures, Journal of the Optical Society of America B, vol. 10, no. 2, 283-295, February 1993. 